\title{
USING ITERA TIVE HUMAN FACTORS METHODS TO ASSESS ACTIVE TRAFFIC MANAGEMENT SIGNING
}

\author{
Mary Anne B. Jeffers ${ }^{1}$, William A. Perez ${ }^{2}$, \& Brian Philips ${ }^{3}$ \\ ${ }^{1}$ Agilex, Chantilly, VA, USA \\ ${ }^{2}$ Leidos, Inc., McLean, VA, USA \\ ${ }^{3}$ Federal Highway Administration, McLean, VA, USA \\ Email: brian.philips@dot.gov
}

\begin{abstract}
Summary: Active traffic management (ATM) is a technique for mitigation of recurrent and non-recurrent congestion. Two ATM tools were evaluated: variable speed limits and lane control signing. An iterative human factors approach included a series of four experiments: a laboratory assessment, a field study, and two dynamic driving simulations. This paper presents the evaluation of signing for one scenario, from among several, to provide an example of the evaluation methodology. That scenario involved closing two lanes on a multi-lane freeway where the exit ramp adjacent to the closed lanes remained open. Results were consistent across experimental settings. The majority of drivers appeared to correctly comprehend the ATM signs in both static and dynamic environments. The combined results from the four experiments showed that the tested ATM signing could achieve about 66 percent driver comprehension and compliance in the presented scenario.
\end{abstract}

\section{INTRODUCTION}

Active traffic management (ATM) involves the mitigation of recurrent and non-recurrent congestion through the use of changeable message signs (CMSs). CMSs are used to display variable speed limit (VSL) and lane control signing (LCS, also known as dynamic lane markings). ATM strategies result in increased peak capacities, smoother traffic flows, and increased safety on major highways (e.g., Lee \& Abdel-Aty, 2008). Various ATM approaches are deployed around the world (Mirshahi et al., 2007).

Although there is an increased interest in using ATM in the U.S., there is no nationwide design standard and limited empirical evidence of how drivers will respond to the CMS messages. In the U.S., Washington and Minnesota recently deployed ATM. However, each location used different approaches for sign structure and content. In Washington, per-lane CMSs were installed on dedicated gantries and adjacent CMSs displayed supplemental information. In Minnesota, perlane CMSs were added to existing sign structures and supplemental CMSs were not provided.

To complement these local efforts, the current work used an iterative process to evaluate ATM signing options from a human factors perspective. One advantage of using this progressive process is that it allows different options to be more quickly and cost effectively assessed, rather than implementing simulation techniques at the start of the project. First, comprehension testing with static CMS images was performed in a laboratory setting. Next, legibility distances were obtained in the field setting. Finally, having down selected the best options from among those evaluated in the laboratory and field settings, driver responses to the ATM messages were 
evaluated in two driving simulator experiments. Each participant was involved in only one individual study so that participant groups did not overlap.

Multiple ATM scenarios were tested across the settings. The scenario presented here involved closing the two right lanes of a multi-lane freeway while the exit ramp (on the right) remained open and available.

\section{METHOD}

\section{Laboratory Setting}

Twenty-six participants (14 males) completed the laboratory study. Participants ranged in age from 20 to 56 years, with a mean age of 36 years. Testing was performed in the FHWA's Sign Laboratory in McLean, VA.

Two variations of the scenario, each with a series of five gantries, were presented. One variation was similar to the Washington deployment (see Figure 1) and the second was similar to the Minnesota deployment (see Figure 2). Participants were tested individually, and instructed to assume they were driving alone and in the left-center lane of a multi-lane highway. One half of the participants viewed the Washington-based series first and the other half viewed the Minnesota-based series first.

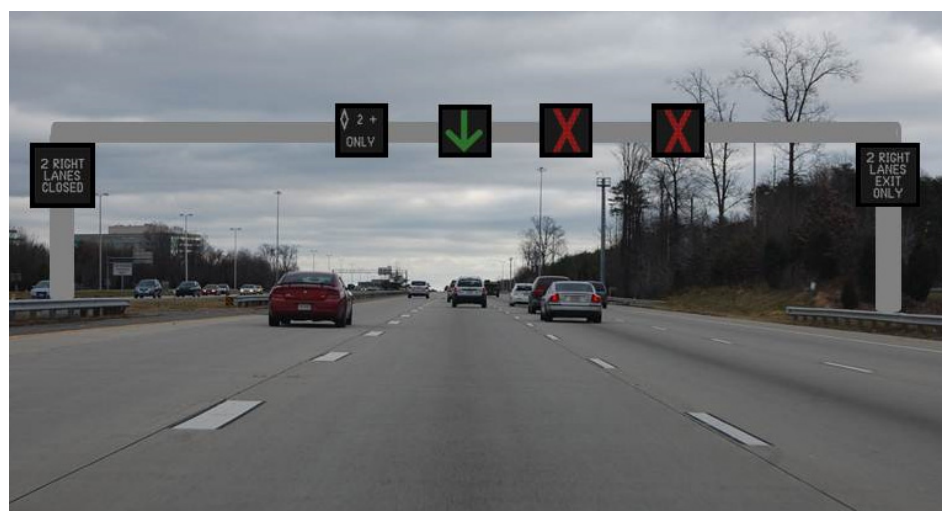

Figure 1. Example laboratory image with structure similar to the Washington deployment

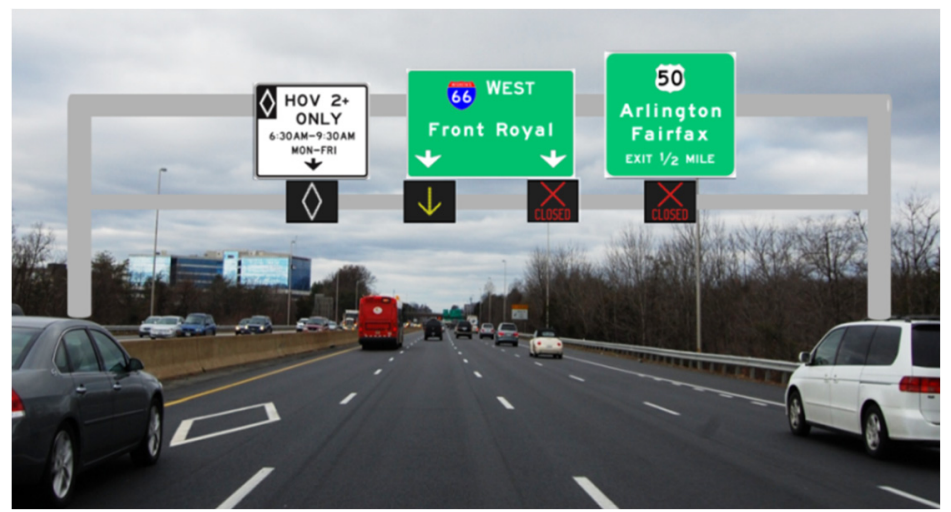

Figure 2. Example laboratory image with structure similar to the Minnesota deployment 
Each series was presented one picture at a time in the order in which they would be encountered on the roadway. For each image in the series, participants were asked "What do the signs mean to you?" and "What action(s) would you engage in based on these signs?" Accuracy of response was stressed over response time.

\section{Field Setting}

Data were obtained from nine participants (six males). The mean age of participants was 39 years (range of 19 to 61 years).

Two ATM signs (red "X" lane closed and yellow "X" lane closing $1 \mathrm{mi}$ ) were tested along with a number of non-ATM specific messages. Both of the ATM images met standards set forth in the Manual on Uniform Traffic Control Devices (MUTCD; Federal Highway Administration, 2009).

Testing was conducted on a $9 \mathrm{~m}$ wide drag strip in Manassas, VA. A CMS was placed on the left side of the drag strip, $376 \mathrm{~m}$ from the start line (see Figure 3). The CMS was a Daktronics VF2320 full color (RGB) matrix display with $20 \mathrm{~mm}$ pixel pitch. The display surface was $1.2 \mathrm{~m}$ by $1.2 \mathrm{~m}$, which accommodated a 64 pixel by 64 pixel display. The brightness of the sign was adjusted for the full daylight test conditions.

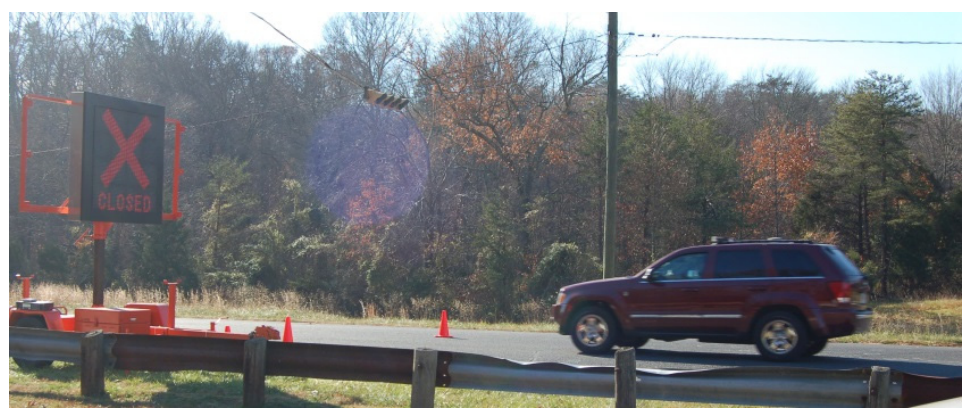

Figure 3. Driver approaching ATM signing in the field setting

Traffic cones were placed on the track to form a $3.6 \mathrm{~m}$ wide lane that curved first from the left to the right side, then back to the left, and ended in the middle of the track at the CMS location. Participants began each trial at the start line. They then briskly accelerated to $40 \mathrm{~km} / \mathrm{h}$ and maintained that speed while they attempted to read the CMS message. Participants were instructed to read the message aloud as soon as possible while still maintaining $40 \mathrm{~km} / \mathrm{h}$ and staying within the marked lane. The dependent measure was the distance from the sign at which the participant finished correctly stating the intended meaning of the sign

\section{First Driving Simulation}

Twenty-four participants (11 males) completed the first driving simulation study. Participants ranged in age from 20 to 79 years, with a mean age of 45 years.

The ATM structure configuration for this simulation (see Figure 4) mirrored the Washington deployment as there was a side-mounted CMS present on the gantries. For this simulation, the HOV restriction was removed after Gantry 1. 




Figure 4. Two right lanes closed scenario design for first driving simulation based on Washington deployment (large rectangle on right represents the side-mounted CMS)

The simulation took place in the Federal Highway Administration's (FHWA) Highway Driving Simulator (HDS). The simulator consisted of a late model compact sedan surrounded by a 240 degree portion of a cylinder (radius of about $3 \mathrm{~m}$ ). Directly in front of the driver, the design eye point of the simulator was about $3 \mathrm{~m}$ from the screen. Stimuli were projected onto the screen by 5 Barco projectors with resolutions of 2,048 horizontal by 1,536 vertical pixels. The car's instrument panel, steering, brake, and accelerator pedal all functioned in a manner similar to realworld compact cars.

Participants drove on a simulated freeway approximately $37 \mathrm{~km}$ in length. Gantries were spaced every $0.8 \mathrm{~km}$ along the roadway. Participants were tasked to follow the highway signs and to take the exit for Holt Avenue. Instructions were to maintain $105 \mathrm{~km} / \mathrm{h}$ and to drive in the second lane from the right, except when passing. Participants were also instructed to follow the ATM signs for lane control guidance. Vehicle traffic was simulated through the VISSIM traffic model to make the driving task more realistic and visually demanding. A short practice session preceded the test session to ensure participants became familiar with the simulator and that they knew they should follow instructions on the ATM signs.

The dependent measures for the first simulation were: (1) whether the participant correctly exited at Holt Avenue, (2) lane choice - measured after passing under each gantry, and (3) average speed - defined as the average speed in two-thirds of the area preceding a gantry through the first third of the area following the structure.

\section{Second Driving Simulation}

Forty-three participants (22 males) completed the second driving simulation study. The mean age was 42 years (range 18 to 76 years). 
The ATM structure configuration for this simulation (see Figure 5) mirrored the Minnesota deployment. Also, in comparison to the first driving simulation, the second simulation began with VSL controls in preparation for the lane closures. However, the position of the closed lanes and destination exit were in the same location (relative to the lane closures) as in the previous experiment.

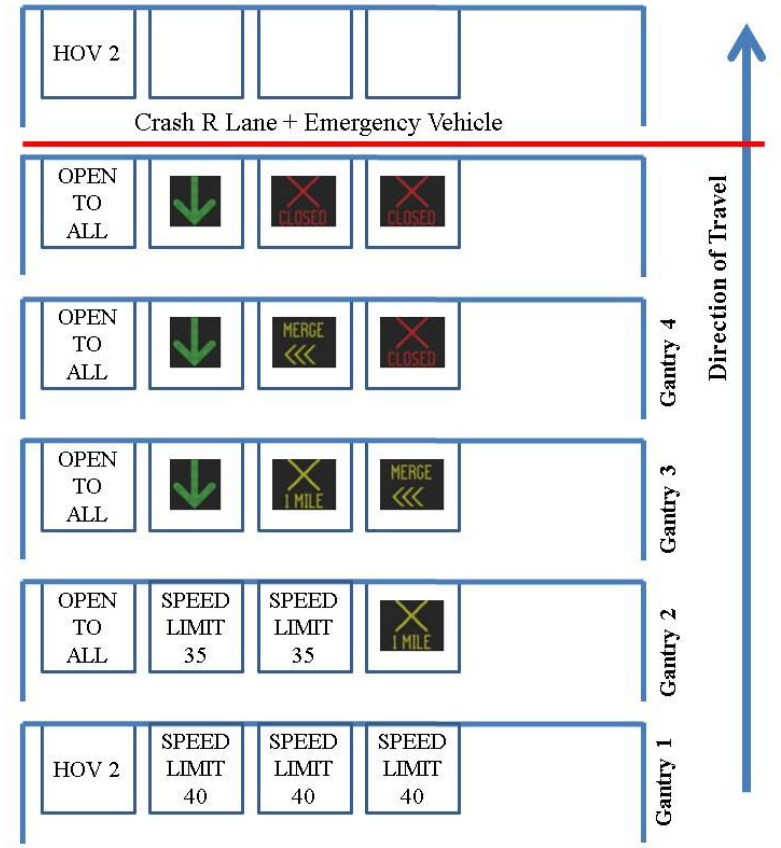

Figure 5. Two right lanes closed scenario design for first driving simulation based on Minnesota deployment

The simulation was also conducted in the FHWA's HDS. However, at the time of the second simulation, the projectors had exceeded their life expectancy and were no longer capable of achieving the necessary brightness or resolution. Therefore, three high-resolution liquid-crystal display (LCD) monitors were mounted to the windshield area of the sedan and used to display the forward 104 degrees of the field-of-view. Each of the LCD displays was $76.2 \mathrm{~cm}$ on the diagonal with a 16:10 aspect ratio and had a resolution of 2,560 horizontal pixels by 1,600 vertical pixels. Two of the original projectors were used to complete the side portions of the 240 degrees horizontal view. As in the first simulation, the car's instrument panel, steering, brake, and accelerator pedal all functioned in a manner similar to real-world compact cars.

The remainder of the methodology for the second simulation was the same as in the first simulation.

\section{RESULTS}

\section{Laboratory Setting}

In general, the results from the laboratory study showed participants correctly interpreted the ATM signs as the signs were presented in sequence for a given scenario. Participants in the study had no previous experience with these types of signs, suggesting the ATM signs were, for the most part, intuitive. Errors included: interpreting advisory VSL signs as regulatory speed limit signs, incorrectly interpreting green overhead guide signs, misinterpretation of the lane closed 
ahead sign with a legend, and confusion over the meaning of the lane open with caution options (both static and flashing).

\section{Field Setting}

Table 1 presents mean legibility distances for the two signs tested in the field. The maximum legibility distance in this test was $381 \mathrm{~m}$. The yellow " $\mathrm{X}$ " had the lowest mean legibility distance and the highest standard deviation. This outcome may have been in part due to the method used to obtain a response because a response did not conclude until the participant completed reading the message. Thus, if a participant struggled in interpreting the nuance in the meaning of the sign, then the response duration would increase and the legibility distance would decrease.

Table 1. Legibility distance measures for selected ATM signs

\begin{tabular}{l|cccc}
\multicolumn{1}{c|}{ Sign } & Mean $(\mathbf{m})$ & STD $(\mathbf{m})$ & Min $(\mathbf{m})$ & Max $(\mathbf{m})$ \\
\hline Yellow "X" MUTCD & 317.14 & 92.99 & 146.30 & 381.00 \\
Red "X" MUTCD & 361.84 & 31.42 & 291.60 & 381.00 \\
\hline
\end{tabular}

\section{First Driving Simulation}

Lane choice was calculated upon passing each of the first three gantries (see Table 2). Chi-square test results indicated there was a significant association between the gantry in the scenario and lane choice; $\chi^{2}(4)=40.20, p<0.001$. Seventy-four percent of the participants successfully exited at Holt Avenue. On average, participants slowed slightly (less than $8 \mathrm{~km} / \mathrm{h}$ ) until they approached the exit and then significantly decreased their speeds (more than $16 \mathrm{~km} / \mathrm{h}$ ) when they began to exit.

Table 2. Distribution of lane choice by gantry for the first simulation study (see Figure 4)

\begin{tabular}{l|cccc} 
& Lane 1 & Lane 2 & Lane 3 & Lane 4 \\
\hline Gantry 3 & $0 \%$ & $52 \%$ & $15 \%$ & $33 \%$ \\
Gantry 2 & $0 \%$ & $48 \%$ & $41 \%$ & $11 \%$ \\
Gantry 1 & $0 \%$ & $4 \%$ & $96 \%$ & $0 \%$
\end{tabular}

\section{Second Driving Simulation}

Lane choice was calculated upon passing each of the first four gantries (see Table 3). Chi-square test results indicated there was a significant association between the gantry in the scenario and lane choice; $\chi^{2}(4)=89.63, p<0.001$. Sixty percent of the participants successfully exited at Holt Avenue. VSL signs were effectively used in this experiment to slow the traffic as they approached the lane closure. By the time participants reached the exit, they were, on average, traveling about $16 \mathrm{~km} / \mathrm{h}$ higher than the posted speed limit on the last VSL sign (shown on Gantry 2) and $32 \mathrm{~km} / \mathrm{h}$ under the posted speed limit $(105 \mathrm{~km} / \mathrm{h})$.

Table 3. Distribution of lane choice by gantry for the second simulation study (see Figure 5)

\begin{tabular}{l|cccc} 
& Lane 1 & Lane 2 & Lane 3 & Lane 4 \\
\hline Gantry 4 & $0 \%$ & $43 \%$ & $12 \%$ & $45 \%$ \\
Gantry 3 & $0 \%$ & $27 \%$ & $56 \%$ & $17 \%$ \\
Gantry 2 & $0 \%$ & $2 \%$ & $95 \%$ & $3 \%$ \\
Gantry 1 & $0 \%$ & $2 \%$ & $95 \%$ & $3 \%$
\end{tabular}




\section{DISCUSSION}

There is a need in the U.S. for design standards and empirical evidence of how drivers respond to various ATM messages. This paper provides an example of an iterative human factors process to meet this need.

Multiple ATM scenarios were tested (one of which was presented in the current paper) across four experiments. Results were fairly consistent across the settings. Drivers comprehended the intended meaning of the signs in both static and dynamic environments. The example scenario was challenging to participants because their target exit was accessible only by using lanes that the CMSs indicated as closed. Most participants comprehended the VSL and LCS in the laboratory. In the driving simulations, the majority of participants correctly accessed the target exit. However, given that about one-third of the drivers chose not to exit, there is still room for improvement in the ATM messages for lane closure scenarios.

The current research used a range of approaches to test different sign options for ATM. Numerous signs were screened using laboratory methods that are suitable for estimating sign comprehension and preference. In addition, field testing used an actual CMS for estimation of legibility distance because high resolution and brightness of the CMS could not be modeled with a laboratory LCD. Use of a subset of ATM signs in the field allowed for the accurate estimation of the legibility distance of these signs. A driving simulator was used to evaluate different sign options under various driving conditions, which allowed the evaluation of responses to the signs in a dynamic environment. The combination of approaches used resulted in assessment of the signs' comprehension, legibility distance, and effect on driver decision making.

\section{ACKNOWLEDGMENTS}

This research was sponsored by the FHWA Office of Operations under contract DTFH61-08-C00006, with guidance from Kevin Sylvester of the MUTCD Team.

\section{REFERENCES}

Federal Highway Administration. (2009). Manual on Uniform Traffic Control Devices for Streets and Highways. Washington, DC: Federal Highway Administration.

Lee, C., \& Abdel-Aty, M. (2008). Testing Effects of Warning Messages and Variable Speed Limits on Driver Behavior Using Driving Simulator. Transportation Research Record: Journal of the Transportation Research Board(2069), 55-64. doi: 10.3141/2069.08

Mirshahi, M., Obenberger, J., Fuhs, C. A., Howard, C. E., Krammes, R. A., Kuhn, B. T., .. . Yung, J. L. (2007). Active Traffic Management: The Next Step in Congestion Management Washington, DC: Federal Highway Administration. 